\title{
Pituitary tumours - a large retrospective single-centre study of over 2300 cases. Experience of a tertiary reference centre
}

\author{
Beata Rak ${ }^{1-3}$, Maria Maksymowicz ${ }^{5}$ Tomasz M. Grzywa ${ }^{6}$, Emir Sajjad ${ }^{2}{ }^{4}$, Monika Pękul ${ }^{5}$, \\ Paweł Włodarski ${ }^{6}$, Grzegorz Zieliński ${ }^{4}$ \\ ${ }^{1}$ Department of Internal Diseases and Endocrinology, Public Central Teaching Hospital, Medical University of Warsaw, Poland \\ ${ }^{2}$ Department of Histology and Embryology, Centre for Preclinical Research, Medical University of Warsaw, Poland \\ ${ }^{3}$ Postgraduate School of Molecular Medicine, Medical University of Warsaw, Poland \\ ${ }^{4}$ Department of Neurosurgery, Military Institute of Medicine, Warsaw, Poland \\ ${ }^{5}$ Department of Pathology and Laboratory Diagnostics, Maria Skłodowska-Curie National Research Institute of Oncology, Warsaw, \\ Poland \\ ${ }^{6}$ Department of Methodology, Centre for Preclinical Research, Medical University of Warsaw, Poland
}

\begin{abstract}
Introduction: Pituitary adenomas (PAs), also known as a pituitary neuroendocrine tumours (PitNET), are usually benign tumours of the anterior lobe of the pituitary gland and account for the third most common intracranial neoplasm. The most common type of pituitary adenoma is lactotroph adenoma, in which dopamine agonists are the first-line treatment. Nevertheless, in selected cases surgery or even radiotherapy may be required. In the current study, we aimed to analyse all patients who underwent surgery due to intrasellar mass in order to evaluate frequency of particular pituitary tumours, clinical diagnosis, and pathology findings.

Material and methods: We retrospectively analysed all cases of patients consecutively operated due to intrasellar mass between $1^{\text {st }}$ January 2010 and $31^{\text {st }}$ December 2018 at the Department of Neurosurgery, Military Institute of Medicine in Warsaw, Poland.

Results: Our database included 2348 cases: 1390 women (59.2\%) and 958 men (40.8\%). The mean age for women was 48.4 years ( $\mathrm{SD} \pm 15.72$; median 49) and for men 50.9 years (SD \pm 14.94 ; median 53). In our cohort we found: 869 gonadotroph and null cell adenomas, 751 somatotroph and mammosomatotroph adenomas, 386 corticotroph adenomas, 71 plurihormonal adenomas, 59 craniopharyngiomas, 44 lactotroph adenomas, 18 purely thyrotroph adenomas, and other rare cases of pituitary tumours including one pituitary carcinoma metastasising to the liver (corticotroph origin).

Conclusions: We provide a comprehensive analysis of both clinical and pathological findings of the largest cohort of patients operated on for pituitary adenomas in one tertiary reference centre. To the best of our knowledge, this is the largest up-to-date published analysis in our country. (Endokrynol Pol 2020; 71 (2): 116-125)
\end{abstract}

Key words: pituitary adenomas; Pas; pituitary neuroendocrine tumours; PitNET; retrospective single-centre analysis

\section{Introduction}

Pituitary adenomas (PAs) are usually benign epithelial tumours of the anterior pituitary gland and account for $10-15 \%$ of all intracranial tumours (in some series reaching over 20\%) with the prevalence estimated at 1/1000 in the general population [1-4]. Pituitary neuroendocrine tumours (PitNET), the new name for pituitary adenomas, was proposed by the International Pituitary Pathology Club in 2017. The new terminology is based on heterogenous biological and clinical spectrum of neoplasm behaviour.

Classification of pituitary adenomas is complex and challenging because it can be performed based on clinical features such as presence or lack of hormonal activity, pituitary insufficiency, visual disturbances, size of the tumour (microadenomas and macroadenomas), radiological findings including suprasellar extension, invasiveness to cavernous sinuses, ultrastructural examination, immunohistochemical evaluation, or according to the most recently introduced classification with the use of transcriptional factors.

Hormonally active (functioning) pituitary adenomas are usually detected earlier compared to non-functioning pituitary adenomas because they secrete hormones to circulating blood thus causing characteristic symptoms for the excess of a particular hormone and may be detected in routine blood tests by endocrinologists. They usually cause significant morbidity and mortality. Classically functioning pituitary 
adenomas (also called hormone secreting) are divided into: prolactinomas (lactotroph adenomas) exhibiting positive immunostaining for prolactin (PRL), somatotroph, mixed GH/PRL, and mammosomatotroph adenomas showing positive immune staining for growth hormone (GH)/GH and PRL, respectively; corticotroph adenomas positive for adrenocorticotropin (ACTH); thyrotroph adenomas positive for thyroid-stimulating hormone (TSH) or gonadotroph adenomas revealing the presence of either single hormone - luteinising hormone (LH), follicle-stimulating hormone (FSH), and alpha subunit of glycoprotein hormones - or a combination of them.

It is worth mentioning that overexpression of hormones within pituitary adenoma detected by immunostaining is not equivocal with the presence of the hormones in circulating blood [5, 6]. About 30\% of non-functioning pituitary adenomas (NFPAs) do not exhibit hormonal staining on IHC, while null cell adenomas account for less than $5 \%$ of them. The most commonly NFPAs are gonadotroph tumours, which was also validated according to the latest classification with the use of transcription factors [7, 8].

Almost three decades ago Knosp et al. introduced a classification aimed at evaluating pituitary tumour invasiveness by the expansion to surrounding tissues - cavernous sinuses [9]. Knosp's classification includes neither the tumours' malignant biology nor any known marker of invasiveness because it was created regarding solely the anatomical range of the tumour. Nevertheless, is still frequently used by radiologists in the assessment of pituitary adenoma invasiveness based on magnetic resonance imaging (MRI). We included data regarding the Knosp scale for cases, in which such information was available.

We report a retrospective analysis of a series of surgically removed (by one neurosurgeon) pituitary tumours over a nine-year period at the Department of Neurosurgery, Military Institute of Medicine in Warsaw (Poland). The presented results were predominantly obtained before the introduction of the 2017 World Health Organisation (WHO) classification and validation of transcriptional factors at our Department of Pathology; therefore, immunohistochemical staining for transcriptional factors was not performed.

\section{Material and methods}

Ethical approval was obtained from the Research Ethics Board at the Medical University of Warsaw for the purpose of our retrospective analysis. The study was performed in accordance with the Helsinki Declaration.

We enrolled in our retrospective analysis all patients operated on for pituitary mass at the Department of Neurosurgery, Military Institute of Medicine (Warsaw, Poland) from $1^{\text {st }}$ January 2010 until $31^{\text {st }}$ December 2018. All patients were clinically diagnosed by lo- cal endocrinologists (from all over the country) and operated by planned surgery due to intrasellar mass by one neurosurgeon G.Z. The subsequent pathological diagnosis was performed by M.M. from the Department of Pathology and Laboratory Diagnostics, Maria Skłodowska-Curie National Research Institute of Oncology (Warsaw, Poland). The pathological evaluation included routine immunohistochemical staining on GH, PRL, ACTH, $\beta$-TSH, $\beta$-FSH, $\beta$-LH, alpha subunit, and Ki-67 for all pituitary adenomas; moreover, in most cases ME was also performed (in every case when the material was preserved). In selected cases, immunostaining was extended to the expression of Cam5.2, sstr2A, sstr5, p53, MGMT, collagen IV, and others, when appropriate.

\section{Immunohistochemistry (IHC)}

Tissue samples were fixed in 10\% buffered formalin, embedded in paraffin, and routinely stained with haematoxylin and eosin. Immunohistochemical staining was performed on paraffin-embedded sections according to the labelled EnVision Flex Visualization System (K8000, Dako/Agilent) with DAB (3,3'-diaminobenzidine) as chromogen, using antibodies available at the time of diagnosis. Currently we are using antibodies against growth hormone $(\mathrm{GH}$, dilution 1:500, cat. no. M5-1328-P1), prolactin (PRL, 1:200, cat. no. M5-1362-P1), ACTH (1:500, cat. no. M5-1452-P1), $\beta$-TSH (1:200, cat. no. M5-1453-P1), $\beta$-FSH (1:500, cat. no. M5-1449-P1), and $\beta$-LH (1:500, cat. no. MS-1448-P1); all antibodies from Thermo Fisher Scientific; the glycoprotein $\alpha$-subunit (dilution 1:1000, cat. no. MCA 1026) from Bio-Rad, UK; TP53 (DO-7; ready to use, cat. no. IR 616), and Ki-67 (MIB-1 clone, ready to use) from Dako; Anti-Cytokeratin (Cam 5.2, cat. no. 790-4555) from Ventana; somatostatin receptors: sstr2A (dilution 1:1500, cat. no. ss800), and sstr5 (dilution 1:1500, cat. no. ss890) from BioTrend.

The cases in which we did not detect positive staining for any of the hormones, we classified as null cell adenomas, and we concluded our final diagnosis based on both IHC and ME results. ME allowed us to distinguish whether "null cell adenoma" classified based on solely IHC staining was indeed true null cell adenoma or had characteristic ultrastructure for the gonadotroph tumours. Thus, our final diagnosis always contributed to the holistic view on the results of both methods.

The staining results of each hormone or hormone's subunit were recorded, and the percentages of immunoreactive cells were graded as follows: $(-)$ all cells negative; $(+)>10 \%$ cells positive.

We classified pituitary adenomas as plurihormonal only if they revealed positive hormonal staining for hormones originating from different lineage backgrounds (for example GH/ACTH, $\mathrm{PRL} / \mathrm{FSH}, \mathrm{TSH} / \mathrm{GH}$, etc.) and the percentage of cells positively stained exceeded $10 \%$. We did not classify as plurihormonal the double contiguous adenomas [10]. What is more, among pituitary adenomas that are classified now as Pit- 1 family, often there is overexpression of subunit alpha in IHC staining. We did not classify tumours exhibiting the solitary additional presence of subunit alpha as a plurihormonal as well. By other authors, they were recently classified as unusual plurihormonal pituitary adenomas [11, 12].

\section{Electron microscopy}

Small pieces of tumour tissues were fixed in $2.5 \%$ glutaraldehyde (SERVA; cat. no. 23116.02), postfixed in $1 \%$ osmium tetroxide (SERVA; cat. no. 31253.02), dehydrated in ethanol and propylene oxide (Sigma-Aldrich, cat. no. 82320-1L), and embedded in Epon (Polysciences, Inc.; Poly/Bed 812, cat. no. 08792-1). Ultrathin sections were counterstained with uranyl acetate and lead citrate followed by examination with a Philips CM120 BioTWIN transmission electron microscope. Corticotroph and somatotroph adenomas were classified on the basis of commonly accepted ultrastructural features, such as densely granulated (DG) or sparsely granulated (SG) 


\section{Statistical analysis}

All statistical calculations were performed using Excel (Microsoft Office), Statistica (StatSoft), or GraphPad Prism 6 (GraphPad Software Inc.). Statistical tests: Chi Mann-Whitney, Kruskal-Wallis, $\mathrm{Chi}^{2}$, or ANOVA were used when appropriate, a cut-off for $\mathrm{p}$-value $=0.05$ was considered statistically significant.

\section{Results}

\section{The general population of patients with pituitary tumours}

We recorded 2348 cases of patients who underwent surgery from $1^{\text {st }}$ January 2010 to $31^{\text {st }}$ December 2018 due to a mass located within the pituitary fossa region. The general population consisted of 1390 women (59.2\%) and 958 men (40.8\%) (Fig. 1). The mean age for women was 48.4 years (SD \pm 15.72 ; median 49 ) and for men 50.9 (SD \pm 14.94; median 53).

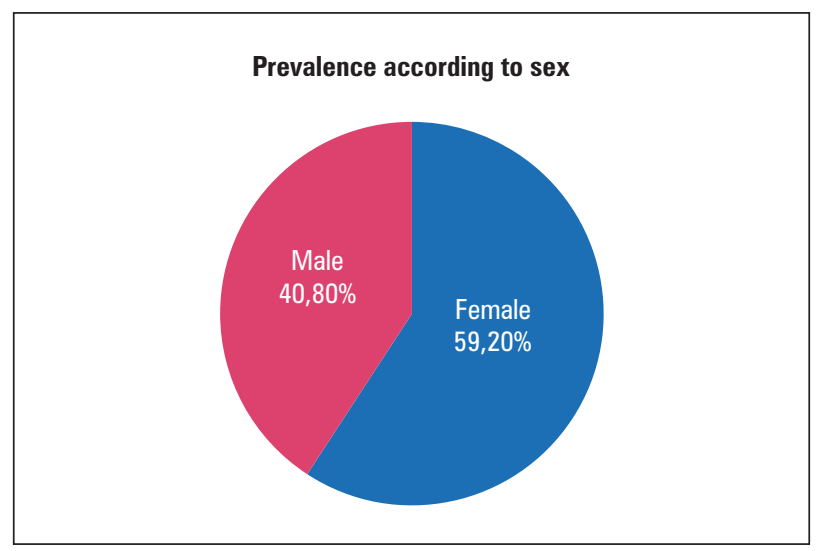

Figure 1. A pie chart shows the ratio of all patients operated to due to mass located within the pituitary fossa region in the years 2010-2018, according to sex
In our series we reported according to presurgical diagnosis (based on clinical, biochemical, and MRI evaluation) the following numbers of cases: 1007 NFPAs, 621 acromegaly, 392 Cushing's disease, 132 prolactinomas, 35 thyrotroph adenomas, and 161 other cases (including 71 craniopharyngiomas, 35 Rathke's cleft cysts, 13 chordomas, and 42 others) (Fig. 2).

\section{Pathology reports}

Nevertheless, histopathological findings revealed some discrepancies, as shown in Figure 3. The final diagnosis based on pathological evaluation was established as listed below: 869 NFPAs (including both gonadotroph and null cell adenomas), 751 somato-

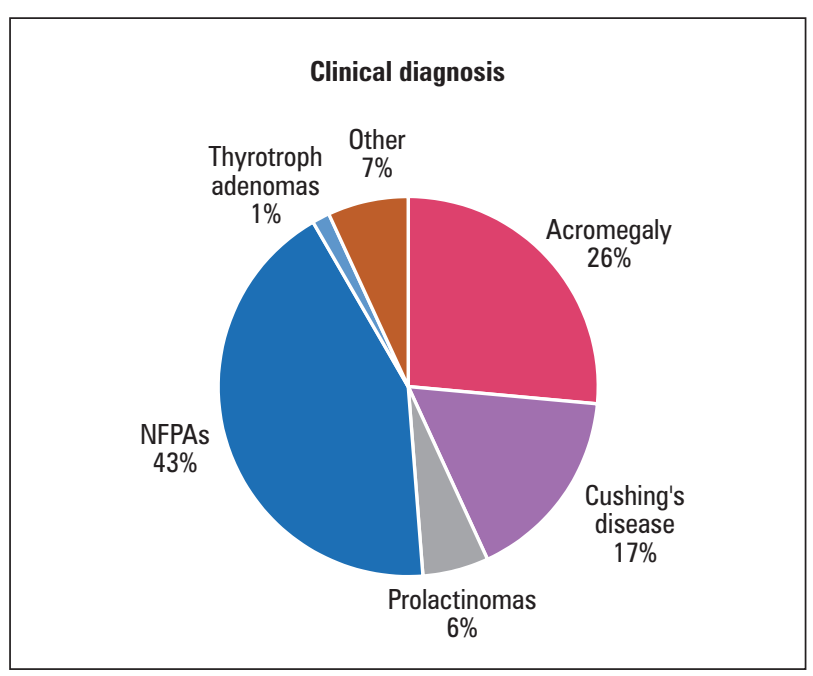

Figure 2. A pie chart shows the ratio of all patients operated to due to mass located within the pituitary fossa region in the years 2010-2018, according to preoperative clinical diagnosis

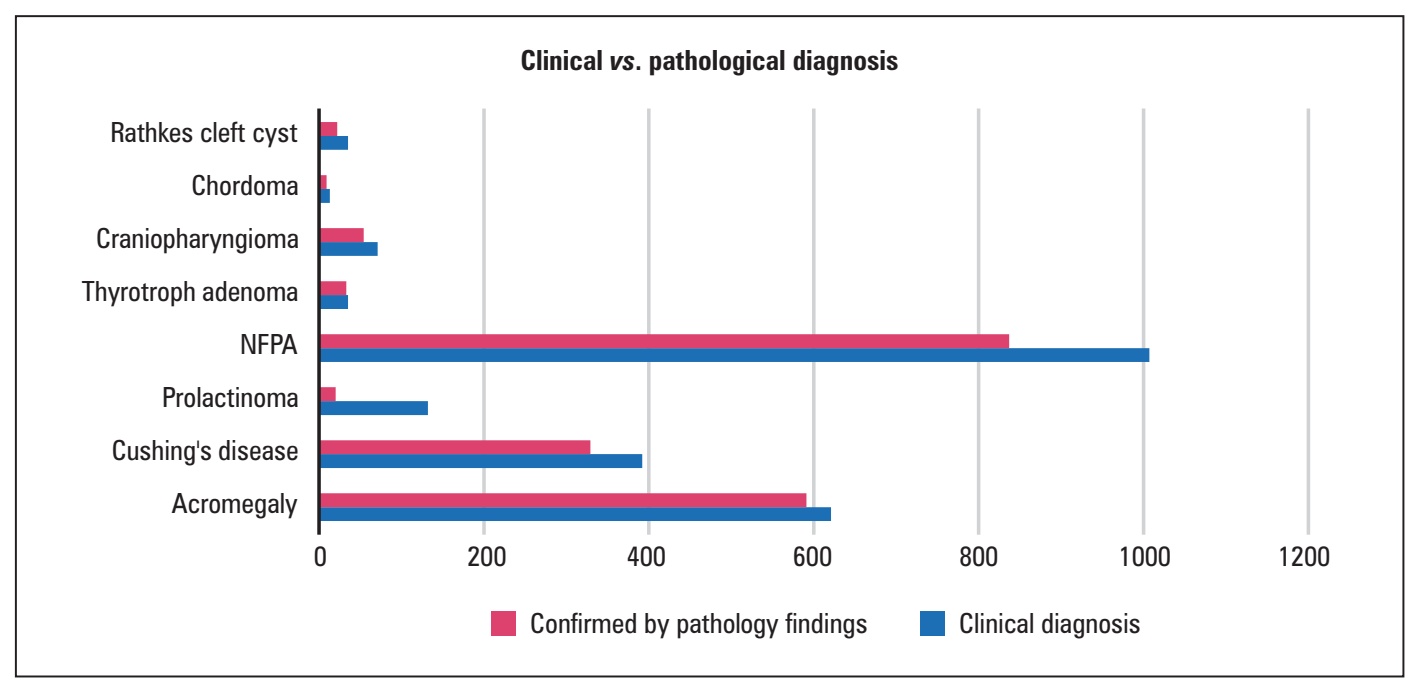

Figure 3. A grouped bar graph shows the numbers of cases for each clinical diagnosis and well-covered diagnosis for those cases confirmed by pathology reports. NFPA — non-functioning pituitary adenoma 


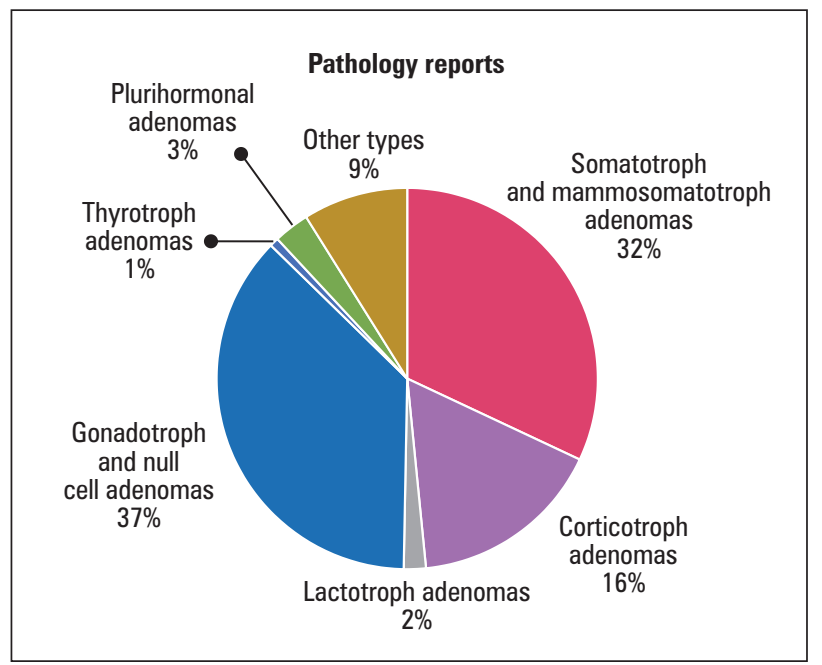

Figure 4. A pie chart shows the percentage of participation of particular tumour types according to histopathological findings. The group 'other types' consists of craniopharyngiomas, Rathke's cleft cysts, and chordomas

troph and mammosomatotroph adenomas together (including silent types), 386 corticotroph adenomas (including silent types and Crooke's cell adenomas), 44 lactotroph adenomas, 18 purely thyrotroph adenomas, 71 plurihormonal adenomas, 59 craniopharyngiomas, 22 Rathke's cleft cysts, nine chordomas, 32 normal pituitaries, and other rare cases. There were 11 double pituitary adenomas in the analysed group; six clearly separated and five contiguous types. In seven patients, somatotroph adenoma coexisted with other adenomas. We recorded only one patient with Cushing's disease and double, clearly separate tumours (coexistence of ACTH-secreting adenoma and PRL-secreting adenoma diagnosed using BIPSS). Percentages of participation for particular types of pituitary tumours are shown in Figure 4.

In our analysis, we recorded four cases of metastases to the pituitary gland $(0.17 \%)$ originating from breast cancers (two patients), thyroid cancer (one patient), and clear cell renal carcinoma (one patient). Other causes of pituitary tumours considered as exceptional findings are shown in Table 1.

During the time of observation, we detected 84 cases of tumour recurrence, with the most prevalent relapse for the corticotroph adenomas (including silent types) reaching 25 cases out of $84(29.76 \%)$. It accounts for an incidence of $6.48 \%$ among corticotroph adenomas.

The majority of NFPAs revealed positive staining for at least one of the hormones or alpha subunits in $92.75 \%$ cases (among them gonadotrophs were predominantly found in 91.6\%), while pure null cell adenomas exhibiting no immunohistochemical staining for hormones or their subunits were found only in 63 cases $(7.25 \%)$.
Table 1. Selected extremely rare causes of tumours located within the pituitary gland, which were collected in our database

\begin{tabular}{ll}
\hline Tumortype & Number of cases \\
\hline Metastasis & 4 \\
\hline Pituicytoma & 4 \\
\hline Germinoma & 2 \\
\hline Epidermal cyst & 2 \\
\hline Astrocytoma pilocyticum & 1 \\
\hline Pineocytoma & 1 \\
\hline Meningioma & 1 \\
\hline Neurocytoma & 1 \\
\hline Osteoblastoma & 1 \\
\hline Osteosarcoma & 1 \\
\hline Arachnoid cyst & 1 \\
\hline Ganglioglioma & 1 \\
\hline Sarcoidosis & 1 \\
\hline Chondrosarcoma & 1 \\
\hline Schwannoma & 1 \\
\hline Glioblastoma & 1 \\
\hline Nasopharyngeal angiofibroma & 1 \\
\hline Pituitary carcinoma & 1 \\
\hline
\end{tabular}

Among PAs exhibiting positive staining for hormones and classified as somatotrophs and corticotrophs we detected also silent subtypes (55 and 38 cases, respectively). We defined silent subtypes according to the literature as tumours without clinical and biochemical signs of the disease, but with apparently present hormonal staining within tumour tissue and characteristic ultrastructure in ME. A graph showing the proportion of the silent types in each group is presented in Figure 10 (attached in the supplementary section). The difference did not reach statistical significance.

Most of the plurihormonal adenomas were hormonally active in regard to clinical diagnosis: only 17 patients were diagnosed as NFPAs, 24 thyrotroph adenomas, 17 acromegaly, eight Cushing's disease, and five lactotroph adenomas.

\section{Age and sex for particular types of pituitary tumours}

Almost in all types of tumours women dominated; the exact distributions of male-to-female ratio for each type of pituitary tumour are presented in Figure 5.

The youngest age of occurrence was observed for lactotroph adenomas in women with a mean age of 34.2 years ( $\mathrm{SD} \pm 12.38$; median 29 ), while for men the mean age was even higher than in the general calculated population, at 55.15 years ( $\mathrm{SD} \pm 15.89$; median 55 ) 


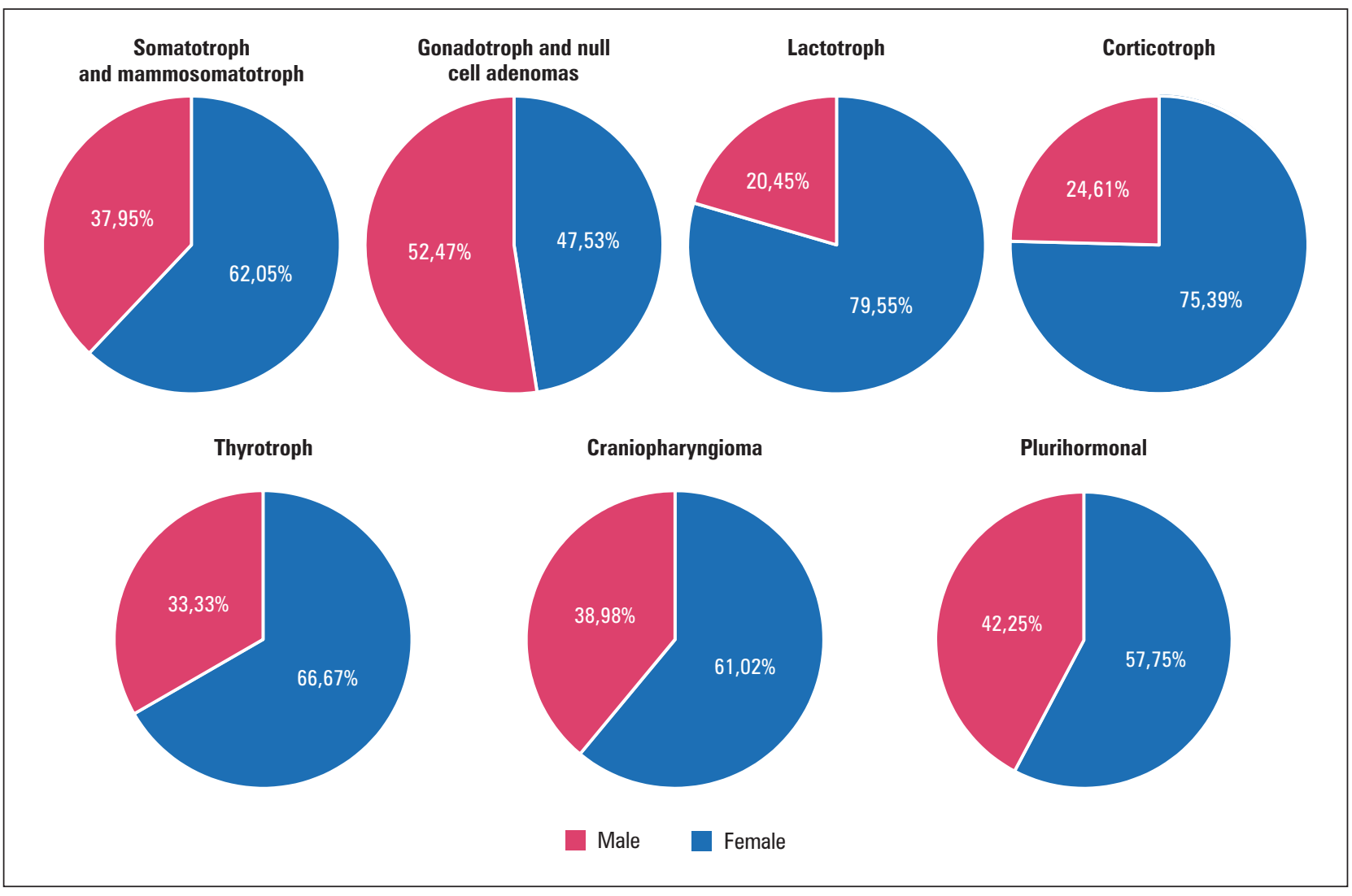

Figure 5. Pie charts showing the distribution of males and females according to pituitary tumour type. Blue colour represents women, and red colour men

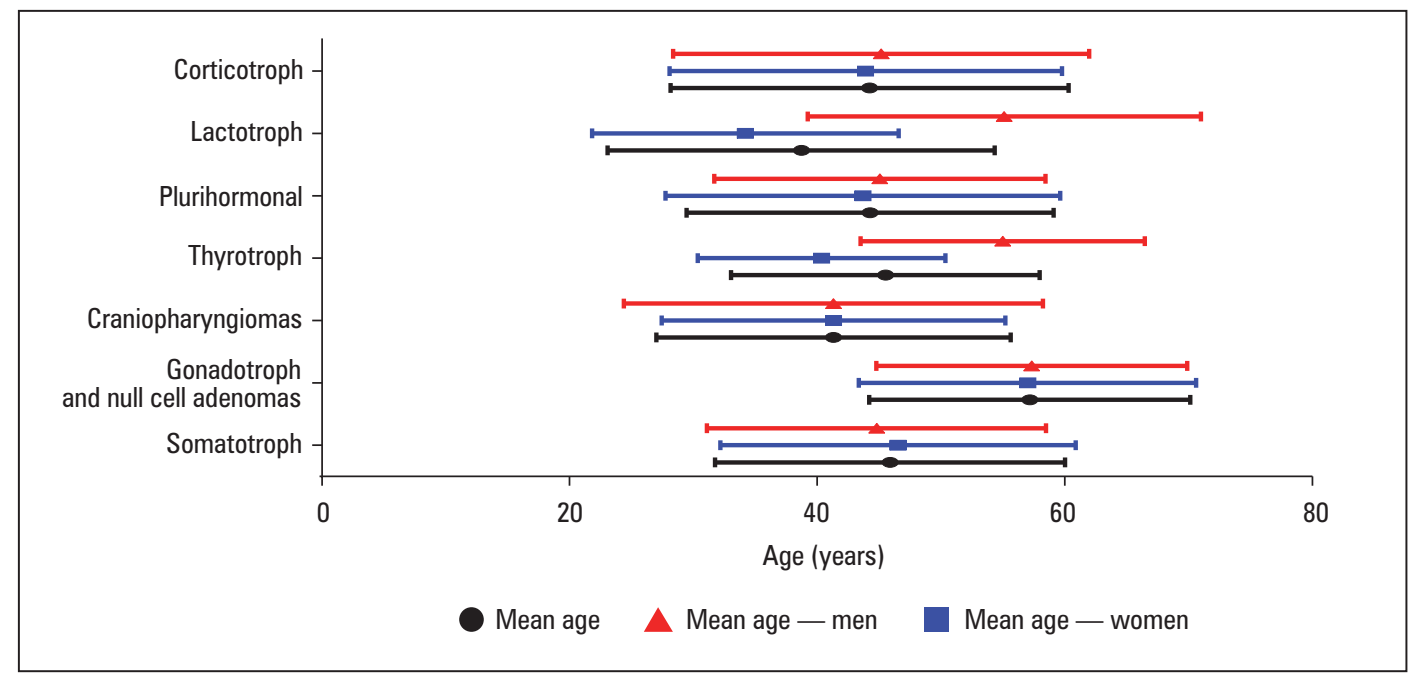

Figure 6. Distribution of age according to pituitary tumour types. Black colour means the mean age for both sexes marked with a dot, horizontal lines reflect the SD value. Blue colour represents woman, and red colour men

compared to 50.9 years (SD \pm 14.94 ; median 53 ), respectively. The total mean age for lactotroph tumours was 38.73 years $(\mathrm{SD} \pm 15.64)$. For acromegaly the mean age was 45.9 (SD \pm 14.14 ; women $44.8 \mathrm{SD} \pm 14.4$, median 47; men 44.8 SD \pm 13.7 , median 45$)$, for gonadotroph and null cell adenomas 57.19 (SD \pm 12.97 ; women 57.02
$\mathrm{SD} \pm 13.62$, median 58; men 57.35 SD \pm 12.56 , median 60 ), for corticotroph adenomas 44.24 (SD \pm 16.09 ; women $43.94 \mathrm{SD} \pm 15.86$, median 40.5; men 45.17 SD \pm 16.8 , median 46), for plurihormonal 44.28 (SD \pm 14.82; women $43.7 \mathrm{SD} \pm 15,95$, median 38 ; men 45.07 $\mathrm{SD} \pm 13.38$, median 41 ), and for craniopharyngiomas 


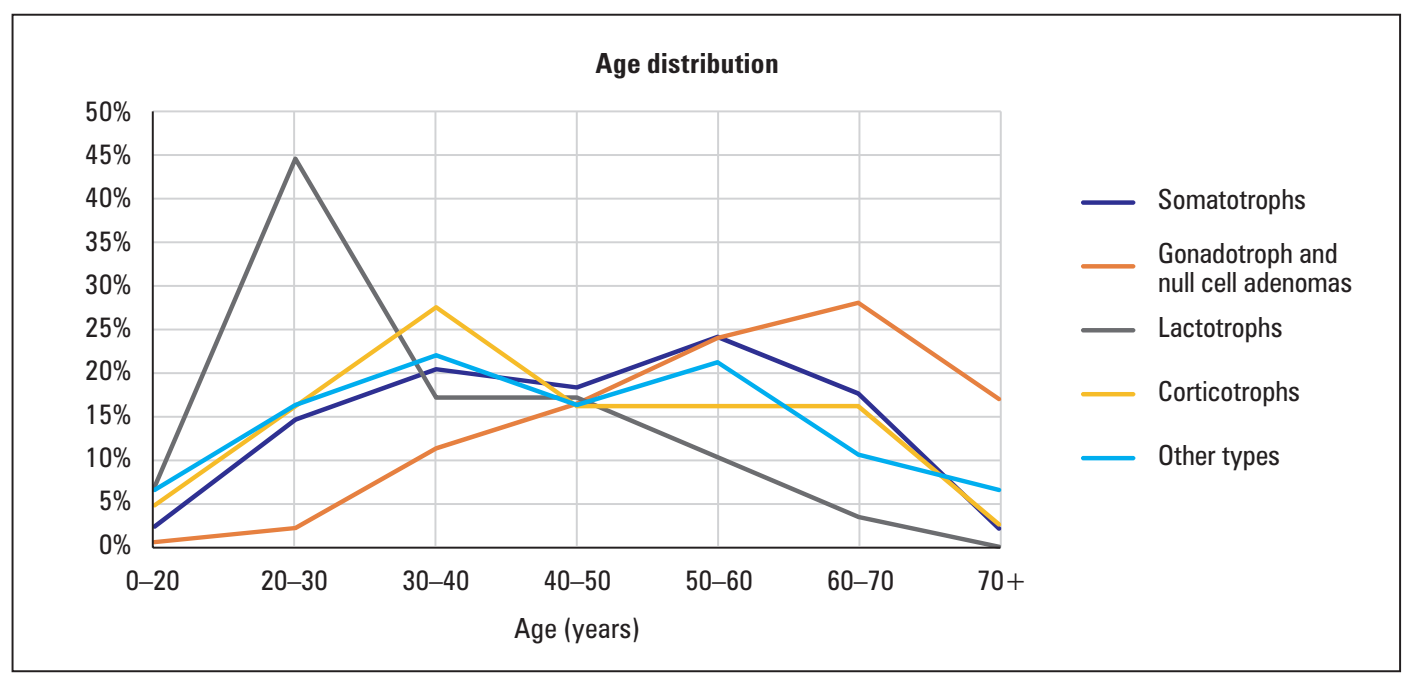

Figure 7. Age distribution (at the time of surgery) for particular types of pituitary tumours. The coloured lines indicate time charts for selected pituitary tumour types: somatotroph adenomas, gonadotroph and null cell adenomas, lactotroph adenomas, corticotroph adenomas, and other types

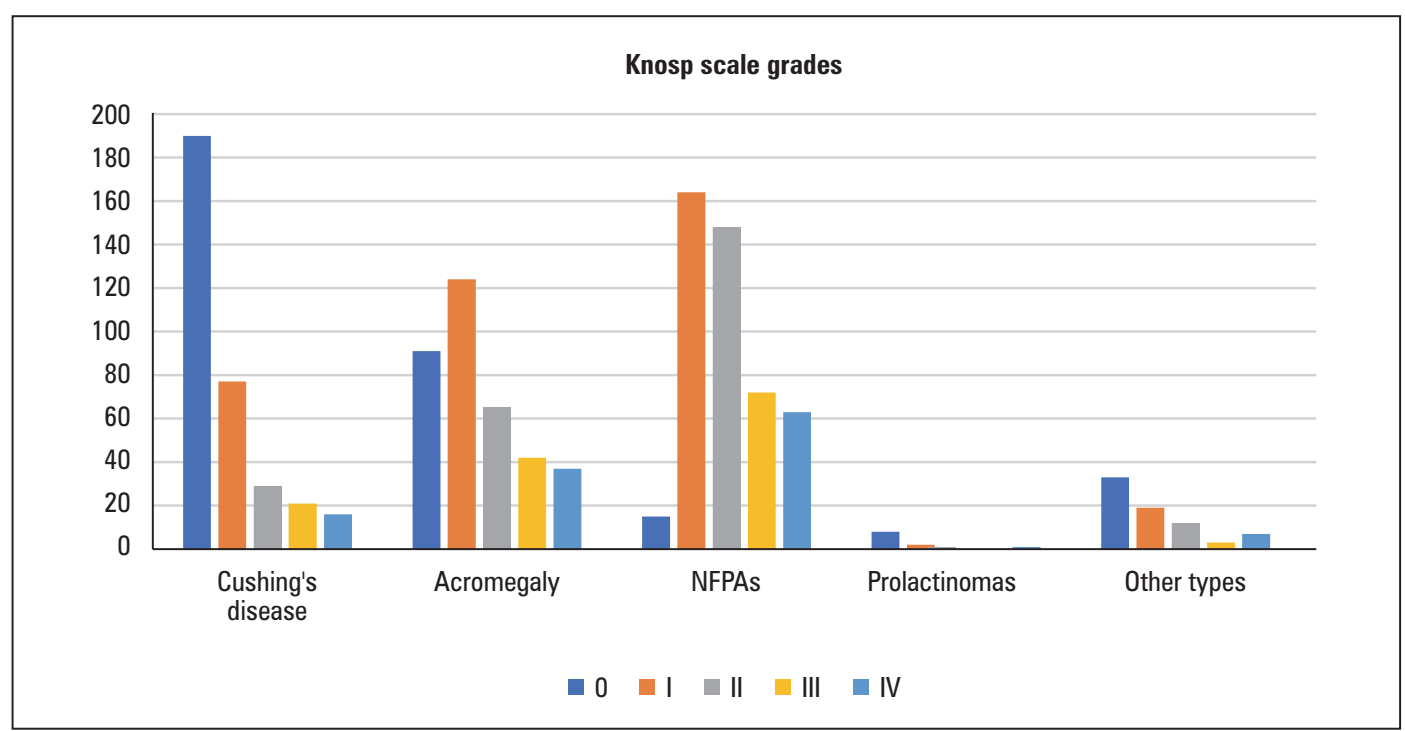

Figure 8. A bar graph illustrates the number of cases for particular tumour types according to Knosp scale grades

41.31 years $(\mathrm{SD} \pm 14.31$; women $41.33 \mathrm{SD} \pm 13.89$, median 38.5; men $41.32 \mathrm{SD} \pm 16.92$, median 41).

A graphical presentation of the age distribution for both sexes (with marked SD values) among particular types of tumours according to pathology reports is shown in Figure 6. The overall age distribution among selected pituitary tumour types is presented in a time chart (see Fig. 7).

\section{Knosp scale grades}

We analysed the frequency of occurrence of particular Knosp scale grades for selected tumour types, as shown in Figure 8, for the cases in which Knosp scale grades were available at the time of data collection. Statistical analysis revealed that those differences were statistically significant between all groups $(p=0.0026)$.

\section{Ki-67 labelling index}

Because most of the cases were collected and diagnosed during the previous classification, which distinguished typical and atypical pituitary adenomas characterised by Ki-67 labelling index $<3 \%$ and $\geq 3 \%$, respectively, we applied this distinction as well. Figure 9 represents the frequency of occurrence typical and atypical pituitary tumours including craniopharyngiomas. The highest Ki-67 labelling index was present for thyrotroph adenomas, somatotroph adenomas, corticotroph adenomas, and gonadotroph and null cell adenomas (16.67\%, 


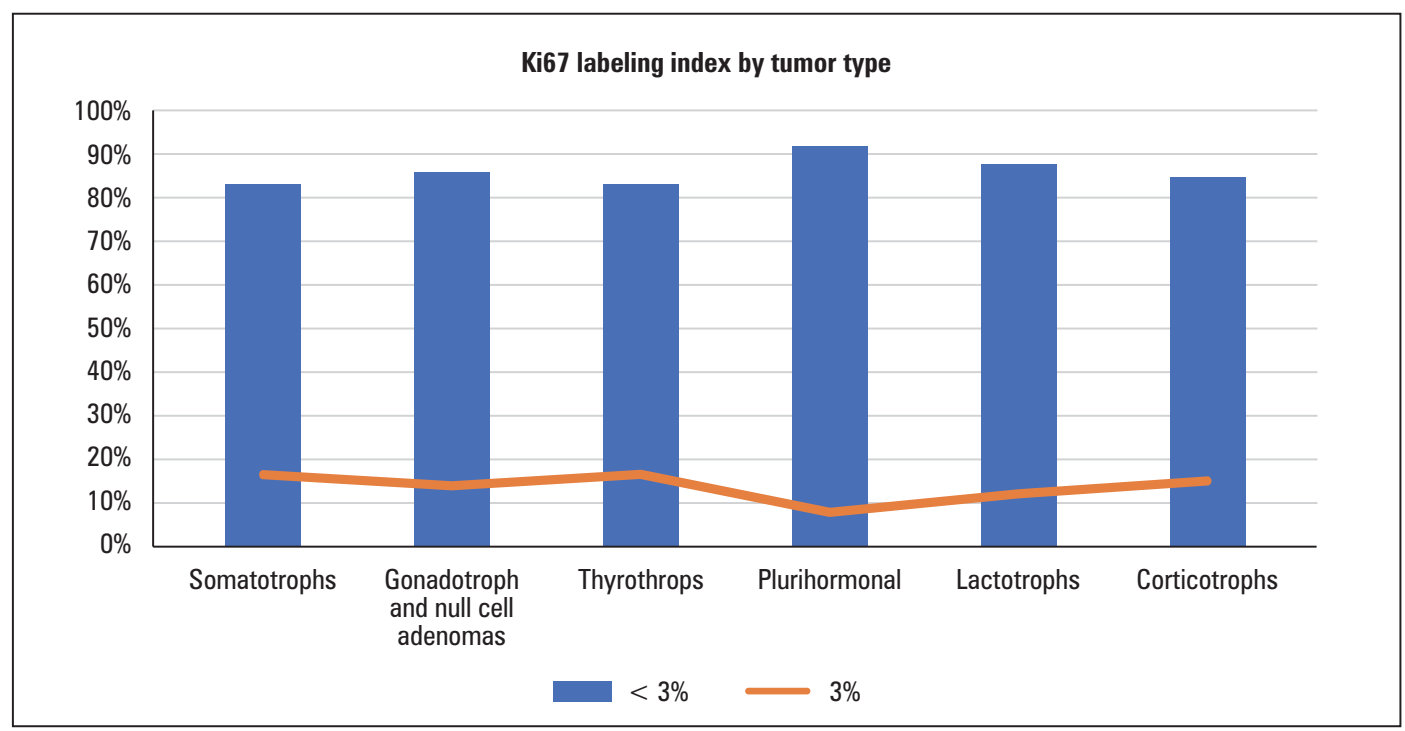

Figure 9. The figure illustrates the Ki-67 labelling index for particular types of pituitary tumours. The Ki-67 labelling indices were grouped into categories: $<3 \%$ and $\geq 3 \%$. The numbers of cases are shown as percentages of participation for selected tumours

$16.59 \%, 15.13 \%$, and $14 \%$, respectively). Plurihormonal adenomas were in the vast majority (over $92 \%$ of cases) characterised by Ki-67 labelling index $<3 \%$.

\section{Discussion}

In our cohort of patients with surgically removed pituitary tumours the most common phenotype was non-functioning pituitary adenoma, and among functioning pituitary adenomas - somatotroph adenoma, which is in accordance with up-to-date published data (Fig. 4) [1, 2, 4]. One needs to remember that in the general population lactotroph adenomas are the most frequent type of PAs. In our study pure prolactinomas accounted only for $2 \%$ of cases, which is due to the fact that the first-line therapy for prolactinomas is an oral administration of dopamine agonists. Neurosurgical intervention is required only for: 1) tumours resistant to pharmacotherapy, 2) medical therapy such CSF leakage, 3) persistent adenoma growth despite pharmacotherapy, 4) pregnancy-related tumour expansion with ophthalmological deficits refractory to medical therapy, and 5) pituitary apoplexy. Among women, lactotroph tumours were diagnosed at the earliest age and the earliest stage (Fig. 6) which is certainly related to symptoms and signs caused by the tumour - infertility and menstruation disturbances.

On the other hand, the reason why prolactinomas were most frequently misdiagnosed in our study is that hyperprolactinaemia found in circulating blood (together with adenoma appearance on MRI) may be caused not solely by the prolactin-secreting tumour, but also as a consequence of a stalk effect. This means that the tumour (for example non-secreting) rises within the pituitary gland or near the stalk and leads to a disrupted connection with the hypothalamus, which interferes with the inhibitory effect of its dopaminergic neurons on lactotroph cells. As a result, pituitary lactotroph cells, which lack the inhibitory influence of hypothalamus, excrete prolactin into circulating blood. Moreover, clinical symptoms including amenorrhoea, decreased libido, galactorrhoea, or headaches and infertility are identical compared with prolactinomas. Nevertheless, it is worth mentioning that in pure prolactinomas the level of prolactin measured in circulating blood correlates with tumour size visible on MRI and is much higher than in pseudoprolactinomas [13-15].

As to other functioning pituitary adenomas, we found in our cohort of patients that acromegaly was the most appropriately diagnosed type of functioning PA $(95.17 \%)$, which was confirmed by pathology reports (Fig. 3). This type of tumour, due to overexpression of growth hormone, leads to increased insulin-like growth factor 1 (IGF-1) level and characteristic clinical and biochemical features. In our research, acromegaly affected most commonly women $(62.05 \%$ vs. $37.95 \%)$ with the mean age of 46.55 years.

Approximately $95 \%$ of acromegaly patients received somatostatin analogues before neurosurgery. The therapy usually led to decreased size of pituitary adenomas, but it did not affect infiltration to cavernous sinuses. The majority of operated cases in which MRI was available were Knosp 0 and Knosp 1 (over $59 \%$ of acromegaly cases).

A study by Park et al. shows that premenopausal women with acromegaly are prone to have larger tu- 
mours with more aggressive tumour types than men [16]. What is more, Larkin et al. reported that histological phenotype of sparsely granulated (SG) somatotroph adenomas, but not genotype, refers more accurately to both clinical and biochemical characteristics as well as response to the octreotide suppression test [17].

In all types of pituitary adenomas apart from NFPAs, women dominated (Fig. 1, 5). In accordance with the literature, our study shows that NFPAs (here: gonadotroph and null cell adenomas) occurred in later decades compared to other PAs independently of sex, with the mean age of 57.19 years (for women 57.02 and for men 57.36) [18-20]. What is more, the majority of NFPAs revealed Knosp scale grades of 2 and more, which accounted for the most advanced stages among all operated pituitary tumours. This fact may be caused by the lack of standard diagnostic laboratory tools that help to select patients affected by NFPAs, because this type of PA may rise silently for many years giving no symptoms or unspecific ones commonly seen by general practitioners such as headaches, nausea, vomiting - until massive enlargement - and then as a macroadenomas they result in visual disturbances by the optic chiasm compression due to suprasellar extension. The most recent study by Németh $\mathrm{K}$ et al. proposed as a useful diagnostic tool monitoring of circulating microRNAs in plasma among patients with PAs including NFPAs [21]. However, this study was performed on a limited number of NFPA patients and without long-term follow up, thus further investigations in this field are crucial.

In our analysis, we recorded only 84 cases of adenomas recurrence and four cases of metastases to the pituitary gland (the most frequent primary origin from breast cancer). Sometimes, complete resection of invasive pituitary tumours is unachievable due to deep infiltration of the cavernous sinus with wrapping around the cavernous part of the internal carotid artery or invasion to adjacent tissues including the skull base. For invasive pituitary adenomas, especially hormonally active tumours, which are resistant to the pharmacological treatment and intractable for surgical intervention, radiotherapy may be introduced [13, 14, 22]. Pituitary carcinomas are extremely rare findings defined by the $\mathrm{WHO}$ as tumours originating from adenohypophyseal cells, having the ability to metastasise either craniospinally or to distant parts of the body (e.g. cervical lymph nodes, lungs, liver) [7]. Although we detected several extremely rare causes of pituitary tumours (see Tab. 1) in our analysis, we recorded only one case of pituitary carcinoma with metastases to the liver in the analysed period - it was arising from Crooke's cell adenoma and is currently treated with temozolomide with a good clinical outcome.
Occasionally, pituitary adenomas elaborate multiple hormones. In our material, we recorded only $3 \%$ of such tumours - called plurihormonal pituitary adenomas - in contrast to other authors, who recorded higher incidences. This fact may be caused by the different methodological approaches used in the laboratories and different meanings of plurihormonality by itself. We relied on the classification mentioned above in the material and methods section, and our plurihormonal group was indeed understood by other authors as unusual plurihormonal pituitary adenomas [11, 12].

It is worth mentioning that these adenomas may be fully functioning, partially functioning (in which only one component is clinically active), or silent. Plurihormonal pituitary adenomas are divided into monomorphous and plurimorphous. Plurihormonal Pit-1-positive adenoma (previously called silent subtype 3 adenoma) is very rare and aggressive tumour that is identified by unique ultrastructural features [23, 24]. Thus, the International Pituitary Pathology Club proposed a new term for pituitary adenoma - pituitary neuroendocrine tumour (PitNET) — in order to highlight their unpredictable behaviour [25].

Multiple pituitary adenomas are composed of double or more separate tumours or two or more different cell types in the same gland. As we showed in results section, we detected several reports of double pituitary adenomas. Their incidence is very low, ranging from 0.4 to $1.3 \%$ in large surgical series, and the aetiology remains ambiguous [26]. Preoperative or intraoperative precise identification of multiple adenomas is crucial to perform an appropriate surgery providing successful outcome [27].

To conclude, herein we analysed nine years of experience of one pituitary neurosurgeon. Our findings are, in general, coherent with up-to-date published reports. It is worth highlighting that, according to the official consensus of the pituitary centres of excellence, pituitary tumour removal performed by an experienced neurosurgeon is associated with fewer complications. It has been established that there exists a volume-outcome effect for pituitary tumours, and the numbers of complications after surgery as well as morbidity and mortality are higher with less experienced surgeons but are significantly lower for neurosurgeons who performed over 200-500 transsphenoidal operations [28-31]. The Pituitary Society and the European Neuroendocrine Association indicated the number of 50 pituitary operations per year as the minimal number for dedicated and experienced pituitary neurosurgeons [32-35]. It is worth noting that our neurosurgeon performed 2348 operations within nine years, giving a mean 260 pituitary surgeries per 
year. To the best of our knowledge, this is the largest retrospective study of surgically removed pituitary tumours to date performed in our country.

\section{Declaration of interest}

The authors have nothing to disclose.

\section{Acknowledgments}

We would like to thank all endocrinologists and other medical specialists who contributed to the study and who took care of patients, whose names would not be possible to mention among the authors for obvious reasons. Especially, we would like to acknowledge professors from different Endocrinology Clinics and Departments across Poland as well as their co-workers.

\section{Funding}

This manuscript was prepared during the internship obtained by the author B.R. as part of financing the doctoral scholarship ETIUDA from the National Science Centre of Poland UMO-2018/28/T/NZ5/00304 and was supported by a grant from the National Science Centre, Poland UMO-2016/23/N/NZ5/02597.

\section{Authors' contributions}

B.R. - study design, literature search, retrospective data collection in the years 2010-2018, initial and final analysis, data interpretation, figure preparation, writing of the whole manuscript; M.M. - performing all pathomorphological evaluations, data collection during the whole study, revising the manuscript critically for important intellectual content; T.G. - retrospective data collection in the years 2010-2017, initial analysis, revising the manuscript; E.S. - data collection, revising the manuscript; MP - assistance in pathomorphological evaluations and data collection during the study; PW — revising the manuscript, supervising the work; G.Z. - performed all neurosurgical operations, data collection during the whole study, revising the manuscript critically for important intellectual content, supervising the work and supporting at each step of the study.

\section{References}

1. Asa S, Ezzat S. The Cytogenesis and Pathogenesis of Pituitary Adenomas*. Endocr Rev. 1998; 19(6): 798-827, doi: 10.1210/edrv.19.6.0350, indexed in Pubmed: 9861546.

2. Daly AF, Rixhon M, Adam C, et al. High prevalence of pituitary adenomas: a cross-sectional study in the province of Liege, Belgium. J Clin Endocrinol Metab. 2006; 91(12): 4769-4775, doi: 10.1210/jc.2006-1668, indexed in Pubmed: 16968795.

3. Daly A, Tichomirowa M, Beckers A. The epidemiology and genetics of pituitary adenomas. Best Pract Res Clin Endocrinol Metab. 2009; 23(5): 543-554, doi: 10.1016/j.beem.2009.05.008, indexed in Pubmed: 19945022.

4. Tjörnstrand A, Gunnarsson K, Evert M, et al. The incidence rate of pituitary adenomas in western Sweden for the period 2001-2011. Eur J Endocrinol. 2014; 171(4): 519-526, doi: 10.1530/EJE-14-0144, indexed in Pubmed: 25084775.

5. Drummond J, Roncaroli F, Grossman AB, et al. Clinical and Pathological Aspects of Silent Pituitary Adenomas. J Clin Endocrinol
Metab. 2019; 104(7): 2473-2489, doi: 10.1210/jc.2018-00688, indexed in Pubmed: 30020466

6. Mete $\mathrm{O}$, Hayhurst $\mathrm{C}$, Alahmadi $\mathrm{H}$, et al. The role of mediators of cell invasiveness, motility, and migration in the pathogenesis of silent corticotroph adenomas. Endocr Pathol. 2013; 24(4): 191-198, doi: 10.1007/s12022-013-9270-y, indexed in Pubmed: 24091601.

7. Nishioka H, Inoshita N. New WHO classification of pituitary adenomas (4th edition): assessment of pituitary transcription factors and the prognostic histological factors. Brain Tumor Pathol. 2018; 35(2): 57-61, doi: 10.1007/s10014-017-0307-7, indexed in Pubmed: 29318396.

8. Nishioka H, Inoshita N, Mete O, et al. The Complementary Role of Transcription Factors in the Accurate Diagnosis of Clinically Nonfunctioning Pituitary Adenomas. Endocr Pathol. 2015; 26(4): 349-355, doi: 10.1007/s12022-015-9398-z, indexed in Pubmed: 26481628.

9. Knosp E, Steiner E, Kitz K, et al. Pituitary adenomas with invasion of the cavernous sinus space: a magnetic resonance imaging classification compared with surgical findings. Neurosurgery. 1993; 33(4): 610-7; discussion 617, doi: 10.1227/00006123-199310000-00008, indexed in Pubmed: 8232800.

10. Zieliński G, Sajjad EA, Maksymowicz M, et al. Double pituitary adenomas in a large surgical series. Pituitary. 2019; 22(6): 620-632, doi: 10.1007/s11102-019-00996-2, indexed in Pubmed: 31598814.

11. Mete O, Cintosun A, Pressman I, et al. Epidemiology and biomarker profile of pituitary adenohypophysial tumors. Mod Pathol. 2018; 31(6): 900-909, doi: 10.1038/s41379-018-0016-8, indexed in Pubmed: 29434339.

12. Lopes MB. The 2017 World Health Organization classification of tumors of the pituitary gland: a summary. Acta Neuropathol. 2017; 134(4): 521-535, doi: 10.1007/s00401-017-1769-8, indexed in Pubmed: 28821944.

13. Melmed S, Casanueva FF, Hoffman AR, et al. Endocrine Society. Diagnosis and treatment of hyperprolactinemia: an Endocrine Society clinical practice guideline. J Clin Endocrinol Metab. 2011; 96(2): 273-288, doi: 10.1210/jc.2010-1692, indexed in Pubmed: 21296991.

14. Eroukhmanoff J, Tejedor I, Potorac I, et al. MRI follow-up is unnecessary in patients with macroprolactinomas and long-term normal prolactin levels on dopamine agonist treatment. Eur J Endocrinol. 2017; 176(3): 323-328, doi: 10.1530/EJE-16-0897, indexed in Pubmed: 28073906.

15. Peverelli E, Treppiedi D, Giardino E, et al. Dopamine and Somatostatin Analogues Resistance of Pituitary Tumors: Focus on Cytoskeleton Involvement. Front Endocrinol (Lausanne). 2015; 6: 187, doi: 10.3389/fendo.2015.00187, indexed in Pubmed: 26733942.

16. Park SeH, Ku CR, Moon JuH, et al. Age- and Sex-Specific Differences as Predictors of Surgical Remission Among Patients With Acromegaly. J Clin Endocrinol Metab. 2018; 103(3): 909-916, doi: 10.1210/jc.2017-01844, indexed in Pubmed: 29272449.

17. Larkin S, Reddy R, Karavitaki N, et al. Granulation pattern, but not GSP or GHR mutation, is associated with clinical characteristics in somatostatin-naive patients with somatotroph adenomas. Eur J Endocrinol. 2013; 168(4): 491-499, doi: 10.1530/EJE-12-0864, indexed in Pubmed: 23288882.

18. Vargas G, Gonzalez B, Ramirez C, et al. Clinical characteristics and treatment outcome of 485 patients with nonfunctioning pituitary macroadenomas. Int J Endocrinol. 2015; 2015: 756069, doi: 10.1155/2015/756069 indexed in Pubmed: 25737722

19. Olsson DS, Bryngelsson IL, Ragnarsson O. Time trends of mortality in patients with non-functioning pituitary adenoma: a Swedish nationwide study. Pituitary. 2017; 20(2): 218-224, doi: 10.1007/s11102-016-0764-8, indexed in Pubmed: 27743172.

20. Asa SL, Ezzat S. Gonadotrope Tumors. Prog Mol Biol Transl Sci. 2016; 143: 187-210, doi: 10.1016/bs.pmbts.2016.08.005, indexed in Pubmed: 27697203

21. Németh K, Darvasi O, Likó I, et al. Comprehensive analysis of circulating microRNAs in plasma of patients with pituitary adenomas. J Clin Endocrinol Metab. 2019 [Epub ahead of print], doi: 10.1210/jc.2018-02479, indexed in Pubmed: 31112271.

22. Greenman Y. Management of endocrine disease: Present and future perspectives for medical therapy of nonfunctioning pituitary adenomas. Eur J Endocrinol. 2017; 177(3): R113-R124, doi: 10.1530/EJE-17-0216, indexed in Pubmed: 28468768.

23. Erickson D, Scheithauer B, Atkinson J, et al. Silent subtype 3 pituitary adenoma: a clinicopathologic analysis of the Mayo Clinic experience. Clin Endocrinol (Oxf). 2009; 71(1): 92-99, doi: 10.1111/j.1365-2265.2008. 03514.x, indexed in Pubmed: 19170710.

24. Horvath E. Fine structural cytology and immunohistochemistry of the non-adenomatous pars distalis of the human pituitary. Pathol Res Pract. 1988; 183(5): 631-633, doi: 10.1016/S0344-0338(88)80028-1, indexed in Pubmed: 3070512

25. Asa SL, Casar-Borota O, Chanson P, et al. attendees of 14th Meeting of the International Pituitary Pathology Club, Annecy, France, November 2016. From pituitary adenoma to pituitary neuroendocrine tumor (PitNET): an International Pituitary Pathology Club proposal. Endocr Relat Cancer. 2017; 24(4): C5-C8, doi: 10.1530/ERC-17-0004, indexed in Pubmed: 28264912. 
26. Kontogeorgos G, Thodou E. Double adenomas of the pituitary: an imaging, pathological, and clinical diagnostic challenge. Hormones (Athens). 2019; 18(3): 251-254, doi: 10.1007/s42000-019-00126-4, indexed in Pubmed: 31388898

27. Ratliff JK, Oldfield EH. Multiple pituitary adenomas in Cushing's disease. J Neurosurg. 2000; 93(5): 753-761, doi: 10.3171/jns.2000.93.5.0753, indexed in Pubmed: 11059654.

28. Ciric I, Ragin A, Baumgartner C, et al. Complications of transsphenoidal surgery: results of a national survey, review of the literature, and personal experience. Neurosurgery. 1997; 40(2): 225-36; discussion 236, doi: 10.1097/00006123-199702000-00001, indexed in Pubmed: 9007854.

29. Barker FG, Klibanski A, Swearingen B. Transsphenoidal surgery for pituitary tumors in the United States, 1996-2000: mortality, morbidity, and the effects of hospital and surgeon volume. J Clin Endocrinol Metab. 2003; 88(10): 4709-4719, doi: 10.1210/jc.2003-030461, indexed in Pubmed: 14557445.

30. Ahmed S, Elsheikh M, Stratton IM, et al. Outcome of transphenoidal surgery for acromegaly and its relationship to surgical experience. Clin
Endocrinol (Oxf). 1999; 50(5): 561-567, doi: 10.1046/j.1365-2265.1999.00 760.x, indexed in Pubmed: 10468920

31. Gittoes NJ, Sheppard MC, Johnson AP, et al. Outcome of surgery for acromegaly--the experience of a dedicated pituitary surgeon. QJM. 1999; 92(12): 741-745, doi: 10.1093/qjmed/92.12.741, indexed in Pubmed: 10581337.

32. Faustini-Fustini M, Pasquini E, Zoli M, et al. Pituitary centers of excellence. Neurosurgery. 2013; 73(3): E557, doi: 10.1227/01.neu.0000431481.25279.37, indexed in Pubmed: 23728454.

33. Schwartz TH. A role for centers of excellence in transsphenoidal surgery. World Neurosurg. 2013; 80(3-4): 270-271, doi: 10.1016/j.wneu.2012.11.019, indexed in Pubmed: 23159660.

34. Bates PR, Carson MN, Trainer PJ, et al. UK National Acromegaly Register Study Group (UKAR-2). Wide variation in surgical outcomes for acromegaly in the UK. Clin Endocrinol (Oxf). 2008; 68(1): 136-142, doi: 10.1 111/j.1365-2265.2007.03012.x, indexed in Pubmed: 17803712.

35. Melmed S, Colao A, Barkan A, et al. Acromegaly Consensus Group. Guidelines for acromegaly management: an update. J Clin Endocrinol Metab. 2009; 94(5): 1509-1517, doi: 10.1210/jc.2008-2421, indexed in Pubmed: 19208732. 